\title{
LOW-FREQUENCY RADIO OBSERVATIONS OF PICOFLARE CATEGORY ENERGY RELEASES IN THE SOLAR ATMOSPHERE
}

\author{
R. Ramesh, K. Sasikumar Raja, C. Kathiravan, and A. Satya Narayanan \\ Indian Institute of Astrophysics, Bangalore 560 034, India; ramesh@iiap.res.in \\ Received 2012 April 21; accepted 2012 November 6; published 2012 December 19
}

\begin{abstract}
We report low-frequency $(80 \mathrm{MHz})$ radio observations of circularly polarized non-thermal type I radio bursts ("noise storms") in the solar corona whose estimated energy is $\sim 10^{21}$ erg. These are the weakest energy release events reported to date in the solar atmosphere. The plot of the distribution of the number of bursts $(d N)$ versus their corresponding peak flux density in the range $S$ to $S+d S$ shows a power-law behavior, i.e., $d N \propto S^{\gamma} d S$. The power-law index $\gamma$ is in the range -2.2 to -2.7 for the events reported in the present work. The present results provide independent observational evidence for the existence of picoflare category energy releases in the solar atmosphere which are yet to be explored.
\end{abstract}

Key words: Sun: activity - Sun: corona - Sun: flares - Sun: radio radiation

\section{INTRODUCTION}

Radio observations are a useful complementary tool for observing signatures of weak, transient energy releases in the solar atmosphere since the related non-thermal emission can easily be detected (see, e.g., Benz 1995). Small-amplitude radio bursts related to microflares (energy $\sim 10^{27} \mathrm{erg}$ ) in the solar atmosphere were first reported by Kundu et al. (1986). Subsequently, several similar events were reported. Recently, Ramesh et al. (2010) presented evidence from low-frequency radio observations for the existence of weak, transient events whose energies were compatible with that of nanoflares (energy $\sim 10^{24} \mathrm{erg}$ ) postulated by Parker (1988). In addition to the aforementioned events, magnetic energy is released in several different forms that are not necessarily related to the traditional definition of flares (Benz 1995; Aschwanden 2004). For example, Katsukawa \& Tsuneta $(2001,2003)$ proposed that the fluctuations in the soft X-rays from active regions are due to ubiquitous tiny bursts of energy releases. The estimated energy of the individual bursts was in the range of $\approx 10^{20}-10^{22} \mathrm{erg}$. The mean value $\left(\approx 10^{21} \mathrm{erg}\right)$ is about $10^{12}$ times weaker than the energy released in some of the strongest flares (energy $\approx 10^{33} \mathrm{erg}$ ) in the solar atmosphere. In view of this, the above weak energy releases were called picoflares (Parnell \& Jupp 2000; Aschwanden et al. 2000; Tsuneta \& Katsukawa 2004). Our interest is to understand these weak energy releases using observations of type I radio bursts (otherwise known as radio noise storms), which constitute the most frequently observed type of solar activity. The emission consists of short-lived $(\sim 0.1-1 \mathrm{~s})$ narrowband radio enhancements (type I or noise storm bursts), superimposed on continuous, slowly varying, long-lasting (for hours to days) broadband background emission called type I or noise storm continuum (see reviews by Elgarøy 1977; Kai et al. 1985). The bursts are considered to be evidence of successive electron accelerations which, unlike the transient acceleration associated with flares, continue for hours or days. It is now generally accepted that the radiation is fundamental $(\mathrm{F})$ plasma emission (Shanmugha Sundaram \& Subramanian 2004) due to the coupling of Langmuir and low-frequency waves (either lower-hybrid or ion-acoustic), and the observed circular polarization results from propagation effects in the presence of a magnetic field. The type I bursts primarily relate to non- flaring sunspot active regions and point to an exceedingly restless corona even during non-flaring times. They are considered to be the signatures of many small steps in coronal evolution, whose cumulative effect is the "gradual" evolution of the corona. The emerging magnetic flux and the changes in the existing active region magnetic fields inevitably lead to field reconnection and type I bursts, which are associated with these small sites of reconnection (Benz \& Wentzel 1981; Spicer et al. 1981).

\section{OBSERVATIONS}

The radio data reported in this work were obtained at $80 \mathrm{MHz}$ on five different observing periods (when there were no temporally associated coronal mass ejections (CMEs), $\mathrm{H} \alpha / \mathrm{X}$-ray flares) during 2011 with the heliograph (Ramesh et al. 1998), and the east-west one-dimensional polarimeter (Ramesh et al. 2008) at the Gauribidanur observatory, ${ }^{1}$ about $100 \mathrm{~km}$ north of Bangalore, India (Ramesh 2011). The coordinates of the array are longitude $=77^{\circ} 27^{\prime} 07^{\prime \prime}$ east and latitude $=13^{\circ} 36^{\prime} 12^{\prime \prime}$ north. The heliograph (Gauribidanur radioheliograph, GRH) is a T-shaped radio interferometer array that produces two-dimensional images of the solar corona with an angular resolution of $\approx 10^{\prime} \times 15^{\prime}$ (R.A. $\times$ decl.) at the above frequency. The antennas (log periodic dipoles) in the array point toward the zenith, and the tilting/steering of the antenna response ("beam") is achieved electronically through the use of diode switches and a cable network. The polarimeter responds to the integrated and polarized flux densities from the whole Sun. The response function ("beam") of the polarimeter array is broad (compared to the Sun) in both right ascension/east-west direction $\left(\approx 4^{\circ}\right.$ at $\left.80 \mathrm{MHz}\right)$ and declination/north-south direction $\left(\approx 90^{\circ}\right)$. This implies that a plot of the data observed with the polarimeter (i.e., the time profile) is essentially the "east-west beam" of the array with amplitude proportional to the strength of the radio emission from the "whole" Sun at the observing frequency/polarization mode, weighted by the antenna gain in that direction. The antennas in the polarimeter array are nonsteerable, unlike the GRH. The radio source(s) responsible for the circularly polarized emission observed with the polarimeter is (are) identified using the two-dimensional radioheliograms

\footnotetext{
1 http://www.iiap.res.in/centers/radio
} 




Figure 1. Gauribidanur polarimeter observations of the type I solar radio bursts in Stokes $I$ at $80 \mathrm{MHz}$ on 2011 February 14 . The integration time is $\approx 256 \mathrm{~ms}$.

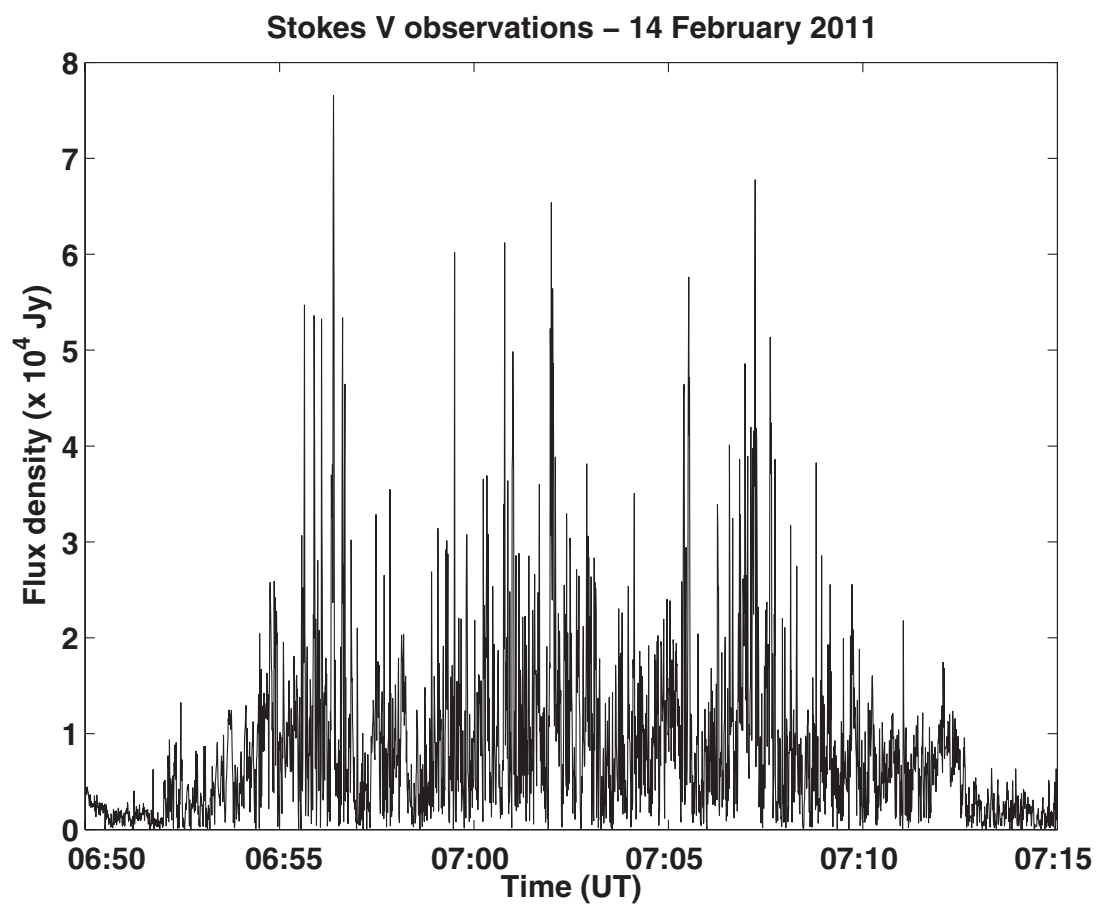

Figure 2. Same as Figure 1, but in Stokes $V$.

obtained with the GRH around the same time. The minimum detectable flux density of the GRH and the polarimeter is $\approx 200$ and $1000 \mathrm{Jy}$, respectively $\left(1 \mathrm{Jy}=10^{-26} \mathrm{Wm}^{-2} \mathrm{~Hz}^{-1}\right.$ ), for an integration time of $\approx 256 \mathrm{~ms}$, and a bandwidth of $\approx 2 \mathrm{MHz}$.

Figures 1 and 2 show the observations with the polarimeter in Stokes $I$ and $V$ on 2011 February 14 during the interval 06:50-07:15 UT, i.e., around the transit of the Sun over Gauribidanur. The limitation in the observing period is mainly due to the non-steerability of the antenna response, and its width $\left(\approx 4^{\circ}\right)$ as mentioned earlier. One can note the presence of numerous short-lived peaks in both the Stokes $I$ and $V$ time profiles. These are characteristic signatures of the type I radio bursts from the solar corona (Elgarøy 1977; Kai et al. 1985). We also independently ascertained the spectral nature of the observed emission as type I radio bursts from online reports. ${ }^{2}$ The peak flux density $(S)$ of the emission is $\approx 14000 \pm 1000 \mathrm{Jy}$ in Stokes $I$. The corresponding degree of circular polarization $\left(\mathrm{dcp}=V_{\text {flux }} / I_{\text {flux }}\right)$ is $50 \%$. Figure 3 shows the time profile of the dep for a few individual bursts. The width of each profile

\footnotetext{
2 ftp://ftp.ngdc.noaa.gov/STP/SOLAR_DATA/SOLAR_RADIO/ BURSTS_Tables/Spectral
} 

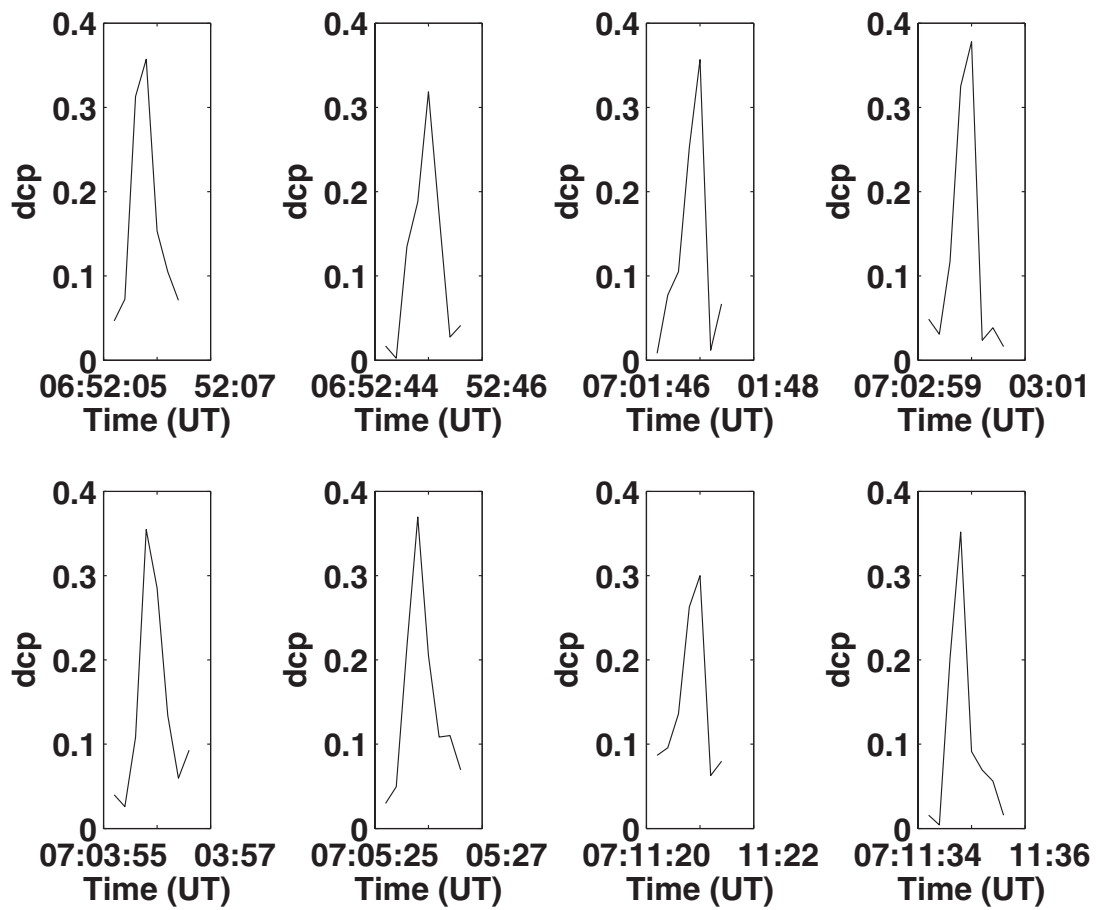

Figure 3. Time profile of the dcp for a few individual type I bursts in Figures 1 and 2 . The time span in each plot is $2 \mathrm{~s}$.

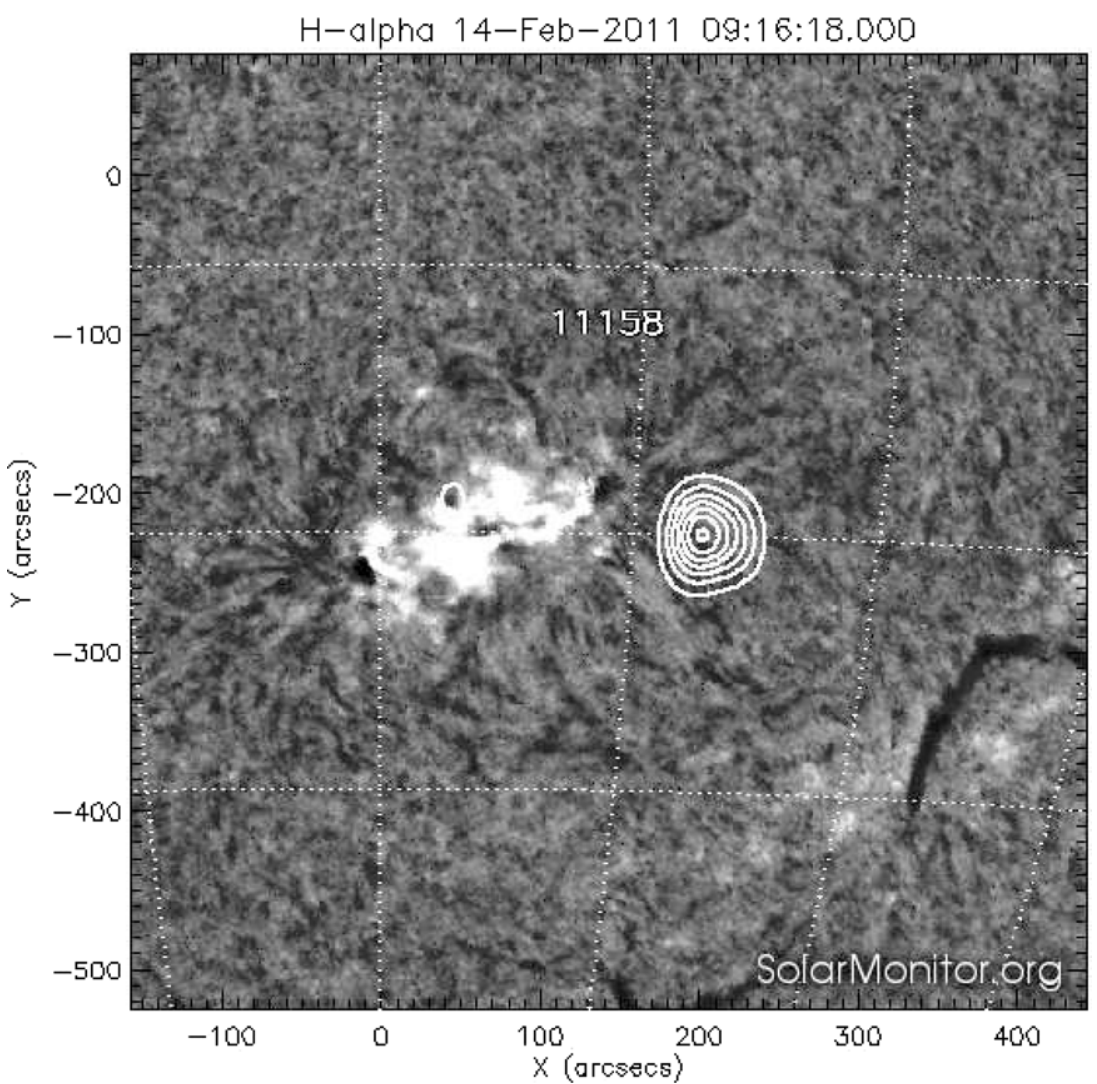

Figure 4. Composite of the $\mathrm{H} \alpha$ image observed at the Big Bear Solar Observatory on 2011 February 14 at $\approx 09: 16$ UT and the GRH radioheliogram (white contours) obtained on the same day at $\approx 07: 00$ UT. The bright $\mathrm{H} \alpha$ emission adjacent to the radio contours is from AR 11158 .

is $\approx 1 \mathrm{~s}$ and the peak dep of the bursts is in the range of $\approx 0.3-0.4$. The information on the location of the radio bursts was inferred from the radioheliogram obtained with the GRH around the same time (Figure 4). Due to the limited angular resolution, only the background noise storm continuum is seen in Figure 4. However, note that the positions of the type I bursts usually scatter over the associated continuum source, and their centroid is within the observed source region of the continuum (Malik \& Mercier 1996). The nearly constant peak dcp of the different bursts mentioned above is probably because 
Table 1

Details of the Type I Radio Bursts Observed with the GRH

\begin{tabular}{|c|c|c|c|c|c|c|c|c|}
\hline $\begin{array}{l}\text { No. } \\
\text { (1) }\end{array}$ & $\begin{array}{l}\text { Date } \\
\text { (2) }\end{array}$ & $\begin{array}{l}\text { Location } \\
\text { (3) }\end{array}$ & $\begin{array}{c}S \\
\left(\times 10^{4} \mathrm{Jy}\right) \\
(4)\end{array}$ & $\begin{array}{c}\mathrm{dcp} \\
\% \\
(5)\end{array}$ & $\begin{array}{c}T_{b} \\
\left(\times 10^{9} \mathrm{~K}\right) \\
(6)\end{array}$ & $\begin{array}{c}W \\
\left(\times 10^{21} \mathrm{erg}\right) \\
(7)\end{array}$ & $\begin{array}{l}\text { Index } \\
(\gamma) \\
(8)\end{array}$ & $\begin{array}{c}B \\
(\mathrm{mG}) \\
(9)\end{array}$ \\
\hline 1 & 2011 Feb 14 & S17W24 & 14 & 50 & 0.8 & 0.5 & -2.3 & 319 \\
\hline 2 & 2011 Mar 12 & N07W90 & 15 & 40 & 0.9 & 0.5 & -2.2 & 354 \\
\hline 3 & 2011 Apr 1 & N37E34 & 7 & 43 & 0.4 & 0.3 & -2.2 & 733 \\
\hline 4 & 2011 Apr 4 & N37W10 & 24 & 50 & 1.4 & 0.9 & -2.2 & 183 \\
\hline 5 & 2011 Jul 30 & N16W18 & 6 & 33 & 0.4 & 0.2 & -2.7 & 950 \\
\hline
\end{tabular}

of this, i.e., the bursts originate from almost the same region of the corona. Observations with the Very Large Array at higher frequency $(333 \mathrm{MHz})$ indicate that the bursts and the continuum are separated by $\approx 1^{\prime}$ (Krucker et al. 1995). We would like to point out here that the noise storm continuum in Figure 4 is not radially above the associated active region but displaced by $\approx 3^{\prime}$. This is because the noise storm sources are usually located in the large-scale coronal loop system connected with an active region, and low-frequency emission comes from the vicinity of the summit of the higher loops (Sheridan et al. 1983; Klein 1998). The average displacement at $80 \mathrm{MHz}$ mentioned in the literature is $\approx 2^{\prime}$ (Dulk \& Nelson 1973). The displacement can be related to the height of the radio emission region in cases where the latter is located near the solar limb (Elgarøy 1977). Assuming the angular size of the type I radio sources to be $\approx 1^{\prime}$ at $80 \mathrm{MHz}$ (Ramesh et al. 2012), we calculated the peak brightness temperature $\left(T_{b}\right)$ of the bursts in Figure 1. The value is $\approx 0.8 \pm 0.1 \times 10^{9} \mathrm{~K}$. The details of the location, peak flux density $(S)$, dcp, and $T_{b}$ for the events reported in the present work are listed in Columns 3-6 of Table 1. The $T_{b}$ values are consistent with the theoretical predictions for type I radio bursts (Melrose 1980). We would like to note here that our above assumption on the source size is justified because (1) Lang \& Willson (1987) and Kerdraon (1979) had earlier reported observations of type I bursts of size of $\approx 0.7$ and $\approx 00^{\prime} .8$ at 328 and $169 \mathrm{MHz}$, respectively and (2) the source size increases with a decrease in frequency (Elgarøy 1977).

\section{RESULTS AND ANALYSIS}

We calculated the energy $(W)$ emitted by the burst source using the relation (Elgarøy 1977),

$$
W=S \Delta t \Delta v R^{2} \Omega e^{\tau}
$$

where $\mathrm{S}$ is the observed flux density at frequency $v, \Delta t$ is the duration of the burst, $\Delta v$ is the bandwidth of the burst, $R \approx 150 \times 10^{9} \mathrm{~m}$ is the Sun-Earth distance, $\Omega$ is the solid angle into which the radio waves are emitted, and $\tau$ is the optical depth at the source at the observing frequency $(v)$. In the present case, $S=14 \times 10^{4} \mathrm{Jy}, \Delta t \approx 256 \mathrm{~ms}$, and $v=80 \mathrm{MHz}$. Assuming $\Delta v=0.02 v \approx 2 \times 10^{6} \mathrm{~Hz}$ (Benz \& Wentzel 1981), which, as mentioned earlier is the same as the bandwidth of the observations in the present case, $\Omega \approx 0.15 \mathrm{sr}$ (Steinberg et al. 1974), and $\tau \approx 3$ at $80 \mathrm{MHz}$ (Ramesh 2005), we get $W \approx 0.5 \pm 0.1 \times 10^{21}$ erg for the 2011 February 14 event (Figure 1). Similar results for the other events in the present work are listed in Column 7 of Table 1. Note that Klein (1995) and Subramanian \& Becker (2004) had estimated the energy contained in the electron population $(\sim 10 \mathrm{keV})$ associated with the noise storm emission to be $\approx 10^{26} \mathrm{erg}$. Therefore, the energy emitted by the burst source mentioned above is probably the lower limit that the acceleration process provides to the radiating electrons (Klein 1995). A comparison of the estimated energies in Table 1 with that compiled by Crosby et al. (1993) and Aschwanden (2004) from observations at other regions of the electromagnetic spectrum indicates that the present values are the weakest energy releases in the solar atmosphere reported to date. These events probably belong to the category of picoflares mentioned earlier. The possibility of fluctuations in the amplitude of the type I bursts in Figures 1 and 2 as well as the other events in Table 1, due to either a screening of the radio source region (by hot and dense evaporated chromospheric material) in the aftermath of flare onset as pointed out by Böhme \& Krüger (1982), Aurass et al. (1990, 1993), and Zaitsev et al. (1994) or formation/deformation of reconnection regions (from where the type I emission is generated) after/before CMEs as shown by Chertok et al. (2001) and Iwai et al. (2012), can be ruled out since the events reported in the present work were not accompanied by either CMEs or $\mathrm{H} \alpha / \mathrm{X}$-ray flares, as mentioned earlier. We verified this using the previously mentioned online resources $^{3}$ and the SGD reports. Also note that (1) according to the results available in the literature, the onset of a flare during an ongoing radio noise storm leads to an overall reduction $(>35 \%)$ in the flux of the latter within a short interval $(\approx 2-3$ minutes $)$ followed by a slow recovery $(\sim \mathrm{hr})$. This is contrary to the present observations where, first of all, there is no reduction in the flux as mentioned above. (2) CME-related variations in the radio flux of the noise storms mentioned in the literature occur over comparatively larger timescales $(\sim$ hrs $)$ unlike the present case, where the changes are over much shorter timescales $(\lesssim 1$ s). Therefore, it is possible that the observed radio emission is likely due to variations in the heating conditions near the source region as described in Li et al. (2009).

It is well known that the distribution of the number of solar flares $(d N)$ in the energy range of $W$ to $W+d W$ follows a powerlaw pattern, i.e., $d N \propto W^{\gamma} d W$, with an index of $\gamma \approx-1.5$ to -1.8 (Drake 1971; Datlowe et al. 1974; Dennis 1985; Crosby et al. 1993). It was pointed out by Hudson (1991) that for weak energy releases $\left(W \lesssim 10^{27} \mathrm{erg}\right)$ in the solar atmosphere, $\gamma$ should be smaller, i.e., $<-2$. Theoretical calculations showing two different power-law indices, $\gamma \approx-1.8$ and -3.5 , respectively, for regular and weak flares in the solar atmosphere were presented by Vlahos et al. (1995) and Georgoulis \& Vlahos (1996). From EUV, UV, and X-ray observations, it was found that $\gamma \approx-2.3$ for events with energies in the range of $\approx 10^{24}-10^{26}$ erg (Porter et al. 1995; Krucker \& Benz 1998; Parnell \& Jupp 2000). Litvinenko (1994) showed that the weak events with $\gamma<-2$ can be explained if the reconnecting current sheet model is assumed for energy accumulation and release. Type I radio bursts are due to small-scale reconnections in the

\footnotetext{
3 www.lmsal.com/solarsoft/latest_events/
} 


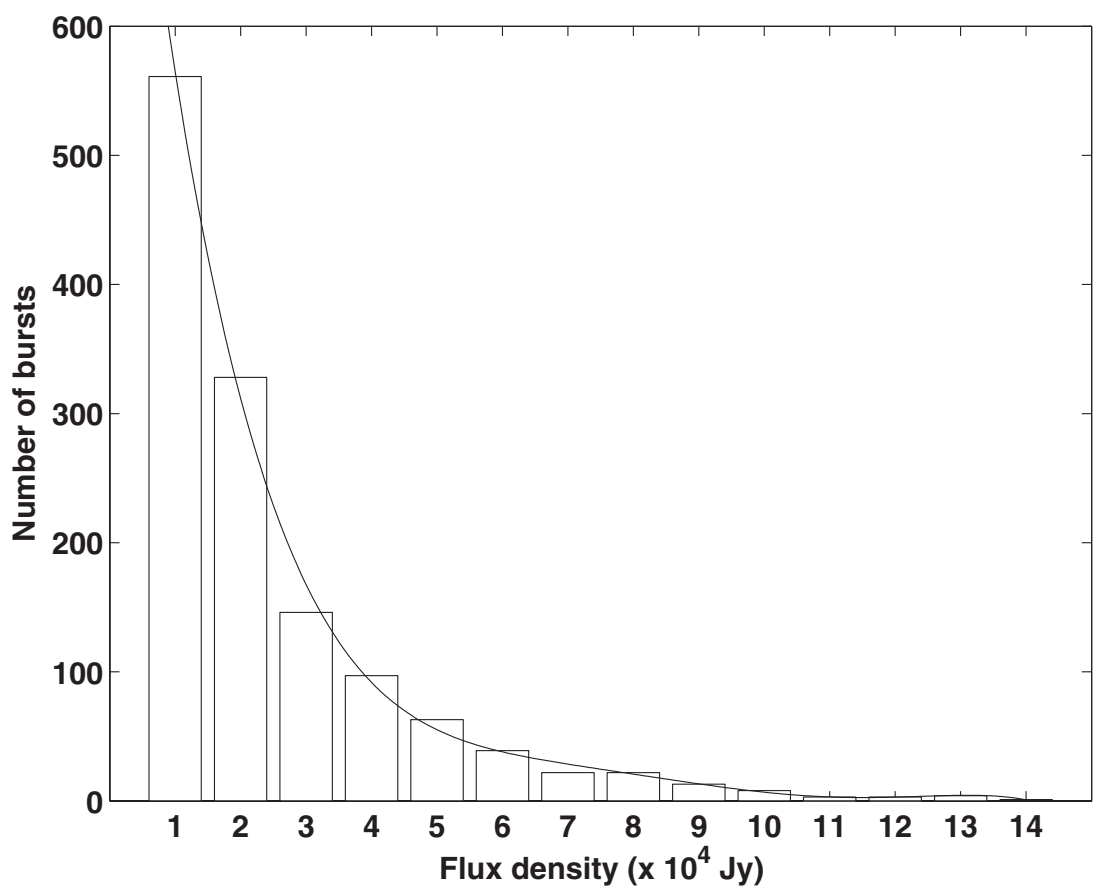

Figure 5. Distribution of the number of type I radio bursts observed on 2011 February 14 (see Figure 1) vs. their corresponding peak flux densities. The index of the power-law fit to the distribution is $\approx-2.3$.

solar atmosphere (as mentioned), and the energy emitted by them is also low (see Column 7 in Table 1). Crosby et al. (1996) suggested that flare-like energy released in the active region is a necessary condition for the onset of type I radio bursts. This prompted us to verify whether the distribution of the number of type I radio bursts $(d N)$ in the flux density range $S$ to $S+d S$ in the present case exhibits a pattern similar to that mentioned above. In Figure 5, we have plotted the number of type I radio bursts observed on 2011 February 14 (see Figure 1) against their peak flux densities in the range $S$ to $S+d S$. One can see that the two quantities vary in a power-law fashion with an index of $\gamma \approx-2.3$. A similar trend was noted for all the other events (see Column 8 in Table 1). We would like to note here that Mercier \& Trottet (1997) reported $\gamma \approx-3$ for the type I bursts they studied at 164, 237, and $327 \mathrm{MHz}$. The flux density of the type I bursts observed by the above authors were $>10^{7} \mathrm{Jy}$. Compared to this, the events reported in the present work are weak (flux density $\sim 10^{4} \mathrm{Jy}$ ).

Assuming the smallest and the largest flare energies estimated from observations thus far to be $W \approx 10^{26} \mathrm{erg}$ and $W \approx 10^{33} \mathrm{erg}$, respectively (Lin et al. 1984; Aschwanden 2004), we calculated the expected power-law index as described in Gary (1998) for weak transient events at the low-energy limit, i.e., $W \approx 10^{21} \mathrm{erg}$. The value is $\gamma \approx-2.6$. The present observational estimates $(\gamma \approx-2.2$ to -2.7$)$ listed in Column 8 in Table 1 are consistent with the above. Following Ramesh et al. (2011), we also calculated the coronal magnetic field $(B)$ near the type I burst location. For the 2011 February 14 event, we found that $B \approx 319 \mathrm{mG}$. Similar values for the other events are listed in Column 9 of Table 1. Note that the contribution to the Stokes $V$ emission from the background corona is expected to be small at $80 \mathrm{MHz}$ (Sastry 2009). Therefore, the tabulated values of $B$ correspond primarily to that of the bursts only.

An inspection of Figure 1 indicates that $\approx 1000$ bursts are present in the total observing period of $\approx 20$ minutes. This gives an occurrence rate of $\approx 1$ burst $\mathrm{s}^{-1}$ which is consistent with the values mentioned in the literature (Benz 1995). Therefore, the power emitted by the type I bursts in the present case is $\approx 10^{21} \mathrm{erg} \mathrm{s}^{-1}$.

\section{SUMMARY}

We have reported observations of weak, circularly polarized, type I radio bursts from the solar atmosphere whose estimated energy $W \sim 10^{21} \mathrm{erg}$. These are the weakest energy release events in the solar atmosphere reported to date. The distribution of the number of bursts $(d N)$ in the flux density range of $S$ to $S+d S$ varies in a power-law fashion with the index $\gamma \approx$ -2.2 to -2.7 . The estimated mean magnetic field strength near the burst source for the events reported in this work is in the range of $\approx 200-900 \mathrm{mG}$. The present observations reinforce the usefulness of low-frequency radio observations for observing weak-energy releases in the solar atmosphere.

Regarding the question of whether or not these weak-energy releases that belong to the picoflare category can contribute to the coronal heating, as pointed out by several authors (Parnell \& Jupp 2000; Tsuneta \& Katsukawa 2004), we would like to note that Book (1981) explained the high temperature of the corona in terms of heating which results from the effect of the emerging magnetic flux on the plasma. Some of his conclusions are as follows. (1) The associated energy is deposited within vertical distances of $\sim 10^{4}-10^{5} \mathrm{~km}$ from the solar surface. For comparison, the typical height at which type I radio emission is observed at frequencies around $80 \mathrm{MHz}$ is $\approx 2 \times 10^{5} \mathrm{~km}$ (Ramesh et al. 2011). (2) The characteristic Alfvén speed $\left(v_{A}\right)$ related to the flux emergence is $\lesssim 500 \mathrm{~km} \mathrm{~s}^{-1}$ (Book 1981; Spicer et al. 1981). Substituting this in the expression $v_{A}=B\left(4 \pi M N_{e}\right)^{-0.5}$, where $N_{e}$ is the electron density and $M=1.9 \times 10^{-24} \mathrm{~g}$ is the mass ascribed to each electron in the plasma (which includes $10 \% \mathrm{He}$ ), we find that the magnetic field $(B)$ at $80 \mathrm{MHz}\left(N_{e}=7.9 \times 10^{7} \mathrm{~cm}^{-3}\right)$ is $\lesssim 2 \mathrm{G}$. Our estimates agree reasonably with this. Evidence has been presented showing that the emerging magnetic flux may provide enough energy to heat the corona by the release of magnetic energy (see, e.g., Schrijver 
et al. 1997). These results suggest a possible connection with the type I noise storms since newly emerging flux has been reported to be one of the causes for the onset of the former. The following reports strengthen the above possibility: (1) Spicer et al. (1981) predicted coronal heating prior to the onset of the type I storms, (2) Melrose (1980) pointed out that the required level of low-frequency turbulence for the generation of type I emission is likely to be present in region where the corona is heated, (3) Raulin \& Klein (1994) noted that the common features in the time profile of soft X-ray brightenings and noise storms point to a physical link between the heating of the plasma in the active region and the noise storm emission. However, from the present observations we find that the power emitted by the type I bursts $\left(\approx 10^{21} \mathrm{erg} \mathrm{s}^{-1}\right)$ is small compared to the energy input for the heating of the corona which is $\approx 10^{27}-10^{28} \mathrm{erg} \mathrm{s}^{-1}$ (Benz 1995; Shimizu 1995). It is possible that type I radio bursts are one of the various weak-energy releases mentioned in the literature (Aschwanden 2004) and all these events together contribute to the heating.

It is a pleasure to thank the staff of the Gauribidanur observatory for their help with observations and maintenance of the antenna and receiver systems there. The $\mathrm{H} \alpha$ image used is courtesy of SolarMonitor.org and the Global High Resolution $\mathrm{H} \alpha$ Network (GHN) team. We thank the referee for helping us to more clearly present the results.

\section{REFERENCES}

Aschwanden, M. 2004, Physics of the Solar Corona: An Introduction (Berlin: Springer)

Aschwanden, M. J., Tarbell, T. D., Nightingale, R. W., et al. 2000, ApJ, 535, 1047

Aurass, H., Böhme, A., \& Karlický, M. 1990, SoPh, 130, 19

Aurass, H., Hofmann, A., Magun, A., Soru-Escaut, I., \& Zlobec, P. 1993, SoPh, 145,151

Benz, A. O. 1995, in Coronal Magnetic Energy Releases, ed. A. O. Benz \& A. Krüger (Lecture Notes in Physics, Vol. 444; Berlin: Springer), 1

Benz, A. O., \& Wentzel, D. G. 1981, A\&A, 94, 100

Böhme, A., \& Krüger, A. 1982, SoPh, 76, 63

Book, D. L. 1981, Comments Plasma Phys. Control. Fusion, 6, 193

Chertok, I. M., Kahler, S. W., Aurass, H., \& Gnezdilov, A. A. 2001, SoPh, 202, 337

Crosby, N., Vilmer, N., Lund, N., Klein, K.-L., \& Sunyaev, R. 1996, SoPh, 167, 333

Crosby, N. B., Aschwanden, M. J., \& Dennis, B. R. 1993, SoPh, 143, 275

Datlowe, D. W., Elcan, M. J., \& Hudson, H. S. 1974, SoPh, 39, 155

Dennis, B. R. 1985, SoPh, 100, 465

Drake, J. F. 1971, SoPh, 16, 152

Dulk, G. A., \& Nelson, G. J. 1973, Proc. Astron. Soc. Aust., 2, 211

Elgarøy, Ø. 1977, Solar Noise Storms (London: Pergamon Press)

Gary, D. E. 1998, in Proc. Nobeyama Symposium, Solar Physics with Radio Observations, ed. T. Bastian, N. Gopalswamy, \& K. Shibasaki (NRO Report 479; Nobeyama: NRO), 129
Georgoulis, M. K., \& Vlahos, L. 1996, ApJL, 469, 135

Hudson, H. S. 1991, SoPh, 133, 357

Iwai, K., Miyoshi, Y., Masuda, S., et al. 2012, ApJ, 744, 167

Kai, K., Melrose, D. B., \& Suzuki, S. 1985, in Solar Radio Physics, ed. D. J. McLean \& N. R. Labrum (Cambridge: Cambridge Univ. Press), 415

Katsukawa, Y., \& Tsuneta, S. 2001, ApJ, 557, 343

Katsukawa, Y., \& Tsuneta, S. 2003, PASJ, 55, 1025

Kerdraon, A. 1979, A\&A, 71, 266

Klein, K.-L. 1995, in Coronal Magnetic Energy Releases, ed. A. O. Benz \& A. Krüger (Lecture Notes in Physics, Vol. 444; Berlin: Springer), 55

Klein, K.-L. 1998, in ASP Conf. Ser. 155, Three-Dimensional Structure of Solar Active Regions, ed. C. E. Alissandrakis \& B. Schmieder (San Francisco, CA: ASP), 182

Krucker, S., \& Benz, A. O. 1998, ApJL, 501, 213

Krucker, S., Benz, A. O., Aschwanden, M. J., \& Bastian, T. S. 1995, SoPh, 160, 151

Kundu, M. R., Gergely, T. E., Szabo, A., Loiacono, R., \& White, S. M. 1986, ApJ, 308,436

Lang, K. R., \& Willson, R. F. 1987, ApJ, 319, 514

Li, B., Cairns, I. H., \& Robinson, P. A. 2009, JGRA, 114, A02104

Lin, R. P., Schwartz, R. A., Kane, S. R., Pelling, R. M., \& Hurley, K. C. 1984, ApJ, 283, 421

Litvinenko, Y. E. 1994, SoPh, 151, L195

Malik, R. K., \& Mercier, C. 1996, SoPh, 165, 347

Melrose, D. B. 1980, SoPh, 67, 357

Mercier, C., \& Trottet, G. 1997, ApJL, 474, 65

Parker, E. N. 1988, ApJ, 330, 474

Parnell, C. E., \& Jupp, P. E. 2000, ApJ, 529, 554

Porter, J. G., Fontenla, J. M., \& Simnett, G. M. 1995, ApJ, 438, 472

Ramesh, R. 2005, in IAU Symp. 226, Coronal and Stellar Mass Ejections, ed. K. P. Dere, J. Wang, \& Y. Yan (Cambridge: Cambridge Univ. Press), 83

Ramesh, R. 2011, in Proc. Astron. Soc. India Conf. Sec. 2, 1st Asia-Pacific Solar Physics Meeting, ed. A. R. Choudhuri \& D. Banerjee (Banglore: ASI), 55

Ramesh, R., Kathiravan, C., Barve, I. V., Beeharry, G. K., \& Rajasekara, G. N. 2010, ApJL, 719, 41

Ramesh, R., Kathiravan, C., Barve, I. V., \& Rajalingam, M. 2012, ApJ, 744,165

Ramesh, R., Kathiravan, C., \& Satya Narayanan, A. 2011, ApJ, 734, 39

Ramesh, R., Kathiravan, C., Sundara Rajan, M. S., Barve, I. V., \& Sastry, Ch. V. 2008, SoPh, 253, 319

Ramesh, R., Subramanian, K. R., Sundara Rajan, M. S., \& Sastry, Ch. V. 1998, SoPh, 181, 439

Raulin, J. P., \& Klein, K.-L. 1994, A\&A, 281, 536

Sastry, Ch. V. 2009, ApJ, 697, 1934

Schrijver, C. J., Title, A. M., van Ballegooijen, A. A., Hagenaar, H. J., \& Shine, R. A. 1997, ApJ, 487, 424

Shanmugha Sundaram, G. A., \& Subramanian, K. R. 2004, ApJ, 605, 948

Sheridan, K. V., Labrum, N. R., Payten, W. J., Nelson, G. J., \& Hill, E. R. 1983, SoPh, 83, 167

Shimizu, T. 1995, PASJ, 47, 251

Spicer, D. S., Benz, A. O., \& Huba, J. D. 1981, A\&A, 105, 221

Steinberg, J. L., Caroubalos, C., \& Bougeret, J. L. 1974, A\&A, 37, 109

Subramanian, P., \& Becker, P. A. 2004, SoPh, 225, 91

Tsuneta, S., \& Katsukawa, Y. 2004, in ASP Conf. Ser. 325, The Solar-B Mission and the Forefront of Solar Physics, ed. T. Sakurai \& T. Sekii (San Francisco, CA: ASP), 289

Vlahos, L., Georgoulis, M., Kluiving, R., \& Paschos, P. 1995, A\&A, 299, 897

Zaitsev, V. V., Aurass, H., Krüger, A., \& Mann, G. 1994, A\&A, 291, 990 